\title{
Lack of evidence for a role of medial temporal lobe structures in visual perception
}

\author{
Larry R. Squire, ${ }^{1,2,3,4,7}$ Yael Shrager, ${ }^{2}$ and Daniel A. Levy ${ }^{5,6}$ \\ ${ }^{1}$ Veterans Affairs Healthcare System, San Diego, California 92161, USA; Departments of ${ }^{2}$ Neurosciences, ${ }^{3}$ Psychiatry, and \\ ${ }^{4}$ Psychology, University of California, San Diego, California 92093, USA; ${ }^{5}$ Department of Neurobiology, Weizmann Institute of \\ Science, Rehovot 76100, Israel, ${ }^{6}$ Gonda (Goldschmeid) Multidisciplinary Brain Research Center, Bar-llan University, \\ Ramat Gan 52900, Israel
}

In a Letter to the Editor in this issue, Bussey et al. (2006) provide additional data from their earlier study to support their idea that the perirhinal cortex is important for visual perception. However, Bussey and colleagues also indicate how their study might nevertheless be understood as a memory impairment rather than as a perceptual impairment. The improved design that they recommend, intended to remove the contribution of memory, is the one that we used in the study that is the subject of their Letter. In our study, and also in a subsequent study, patients with extensive medial temporal lobe lesions, as well as patients with limited hippocampal lesions, were intact in all tests of visual perception. At this time, there is a lack of evidence that the medial temporal lobe is involved in visual perception. In contrast, there is compelling evidence, accumulated over more than four decades, that the medial temporal lobe is important for memory.

The idea that medial temporal lobe structures are principally important for memory has a long history, beginning with the first descriptions of the noted patient H.M. (Scoville and Milner 1957). H.M. became profoundly amnesic after sustaining a large bilateral medial temporal lobe resection for the relief of epilepsy, but his intellectual and perceptual functions remained intact (Milner et al. 1968). A new proposal is that the perirhinal cortex within the medial temporal lobe is important for visual perception, as well as memory, especially for discriminating among stimuli that have high "feature ambiguity" (see Bussey et al. 2002 , 2003). This idea has been based mainly on work with nonhuman primates. Yet, in work with experimental animals it is difficult to distinguish impairments in visual perception from impairments in learning and memory because the tests used to assess perceptual ability typically require training. Indeed, many of the findings that have been taken to reflect impaired visual perception in monkeys with perirhinal lesions have been questioned on the grounds that the findings can be readily explained as reflecting memory impairment (Hampton 2005).

With this background, one study (Bussey et al. 2003, Experiment 2) is of special interest, because it was designed explicitly to disentangle learning effects from perceptual performance. Monkeys first learned easy discrimination problems and then were given transfer tests with the original discriminanda blended together to create feature-ambiguous stimuli. Monkeys with perirhinal lesions were impaired on the transfer tests.

Because the transfer tests involved blocks of 32 trials, during which learning could potentially have occurred, it has been natural to wonder what performance was like during the first few trials (Hampton 2005; Levy et al. 2005). The data presented in their Letter (Bussey et al. 2006) suggest that a deficit was in fact apparent in the first block of four trials (though it is not stated whether the deficit was statistically significant). In any case, the

\section{${ }^{7}$ Corresponding author.}

E-mail Isquire@ucsd.edu; fax (858) 552-7457.

Article and publication are at http://www.learnmem.org/cgi/doi/10.1101/ Im.178406. data appear consistent with a failure in perceptual generalization. $^{8}$

These results are somewhat surprising because Hampton and Murray (2002) carried out a related study and found that following the learning of discrimination pairs, monkeys with perirhinal lesions performed normally on interpolated trials in which the stimuli were rotated, enlarged, shrunk, presented without color, or degraded with masks. That is, perceptual generalization was fully intact. In that study, though, feature ambiguity was not explicitly manipulated.

In their Letter, Bussey et al. suggest a possible way to understand their data in terms of a memory impairment. They suggest that, when the transfer pairs were presented, monkeys with perirhinal lesions might have been disadvantaged by trying to compare the new (transfer) stimuli to a stored memory of the correct (unblended) stimulus that had originally been learned. This possibility only emphasizes how difficult it is to test perceptual performance in animals in the absence of any burden on learning and memory. And it suggests that more work is needed with experimental animals if a case is to be made that perirhinal lesions impair perception. As outlined by Hampton (2005), most existing studies are flawed by the confounding of perception and memory.

Bussey et al. (2006) recommend an improved design, where the original target stimulus is presented together with the blended (or morphed) stimuli so that memory for the target stimulus is not required to perform the task. This is precisely what was done in Levy et al. (2005), the work to which the Letter by Bussey et al. is directed. Levy et al. (2005) tested profoundly amnesic patients with well-characterized medial temporal lobe lesions that include all of the perirhinal cortex. The patients were given a number of tasks involving the same kind of featureambiguous stimuli used by Bussey et al. (2003). Two of the tasks eliminated the requirement to learn about the test items. One task (Levy et al. 2005, Experiment 2a) involved same-different judgments with trial-unique stimuli. Another task (Levy et al. 2005, Experiment 2b) used the improved design that Bussey et al. (2006) recommend in their Letter. Visual perception was fully intact in both these experiments.

Bussey et al. (2006) also point to a recent study in humans that incorporated the improved design that they recommend

\footnotetext{
${ }^{8}$ Bussey et al. (2006) also write that their results from 2003 are opposite to our earlier findings that medial temporal lobe lesions impair performance during the early trials of simple, rapidly acquired discriminations. However, their monkeys with perirhinal lesions appeared disadvantaged in the early trials of learning easy discriminations. As estimated from Bussey et al. (2003, Fig. 5B), monkeys with perirhinal lesions scored $55 \%, 67 \%$, and $77 \%$ correct for trials $1-8$ $9-16$, and $17-24$ vs. $52 \%, 81 \%$, and $91 \%$ correct for controls (compare their results to the similar results from Buffalo et al. 1999, Fig. 10A; Teng et al. 2000, Fig. 2). A straightforward explanation of why such an impairment occurs in both humans and nonhuman primates during the learning of simple discriminations appears in Zola and Squire (2000).
} 
(Lee et al. 2005). Lee et al. (2005) reported that patients with lesions of the medial temporal lobe were impaired on high feature-ambiguity discriminations between morphed faces, objects, and scenes and that patients with hippocampal lesions were impaired in discriminating between morphed scenes (interpreted as impaired spatial perception). We recently carried out a similar study of two patients with large medial temporal lobe lesions and four patients with limited hippocampal lesions (Shrager et al. 2006). We used the same two tasks used by Lee et al. (2005), along with two additional tasks. The patients had intact visual perception in all tasks.

The impairments reported by Lee et al. (2005) may reflect the fact that their tests involved items that repeated from trial to trial, so that controls might have benefited from learning. More important, it is noteworthy that (1) three of the seven patients had lesions that were described as lying outside the anatomical boundaries that were supposed to define the groups, and (2) the lesions were characterized by visual ratings of magnetic resonance images that left large amounts of brain tissue unexamined. For example, for the lateral temporal lobe, Lee et al. (2005) rated a single coronal section on a $0-3$ scale, while we performed quantitative volumetric analysis at 1-mm intervals rostro-caudally and measured all the coronal sections in which the lateral temporal lobe was present (mean $=58$ sections). Careful neuroanatomical descriptions of patients are of paramount importance as hypotheses about medial temporal lobe functions become more specific and tied to particular structures. At the present time, there is no compelling evidence to suppose that the perirhinal cortex is involved in object perception or that the hippocampus is involved in spatial perception. However, visual perceptual impairments do occur when damage extends more laterally into the temporal lobe. Damage restricted to the medial temporal lobe impairs memory.

\section{Acknowledgments}

The work from our laboratory discussed here was supported by the Medical Research Service of the Department of Veterans Affairs, NIMH Grant 24600, and the Metropolitan Life Foundation.

\section{References}

Buffalo, E.A., Ramus, S.J., Clarke, R.E., Teng, E., Squire, L.R., and Zola, S.M. 1999. Dissociation between the effects of damage to perirhinal cortex and area TE. Learn. Mem. 6: 572-599.

Bussey, T.J., Saksida, L.M., and Murray, E.A. 2002. Perirhinal cortex resolves feature ambiguity in complex visual discriminations. Eur. J. Neurosci. 15: 365-374.

. 2003. Impairments in visual discrimination after perirhinal cortex lesions: Testing 'declarative' vs. 'perceptual-mnemonic' views of perirhinal cortex function. Eur. J. Neurosci. 17: 649-660.

. 2006. Perirhinal cortex and feature-ambiguous discriminations. Learn. Mem. (this issue).

Hampton, R.R. 2005. Monkey perirhinal cortex is critical for visual memory, but not for visual perception: Re-examination of the behavioural evidence from monkeys. Q. J. Exp. Psychol. 58B: 283-299.

Hampton, R.R. and Murray, E.A. 2002. Learning of discriminations is impaired, but generalization to altered views is intact, in monkeys (Macaca mulatta) with perirhinal cortex removal. Behav. Neurosci. 116: $363-377$.

Lee, A.C., Bussey, T.J., Murray, E.A., Saksida, L.M., Epstein, R.A., Kapur, N., Hodges, J.R., and Graham, K.S. 2005. Perceptual deficits in amnesia: Challenging the medial temporal lobe 'mnemonic' view. Neuropsychologia 43: 1-11.

Levy, D.A., Shrager, Y., and Squire, L.R. 2005. Intact visual discrimination of complex and feature-ambiguous stimuli in the absence of perirhinal cortex. Learn. Mem. 12: 61-66.

Milner, B., Corkin, S., and Teuber, H.-L. 1968. Further analysis of the hippocampal amnesic syndrome: 14-year follow-up study of H.M. Neuropsychologia 6: 215-234.

Scoville, W.B. and Milner, B. 1957. Loss of recent memory after bilateral hippocampal lesions. J. Neurochem. 20: 11-21.

Shrager, Y., Gold, J.J., Hopkins, R.O., and Squire, L.R. 2006. Intact visual perception in memory-impaired patients with medial temporal lobe lesions. J. Neurosci. (in press).

Teng, E., Stefanacci, L., Squire, L.R., and Zola, S.M. 2000. Contrasting effects on discrimination learning after hippocampal lesions and conjoint hippocampal-caudate lesions in monkeys. J. Neurosci. 20: $2853-2863$.

Zola, S.M. and Squire, L.R. 2000. The medial temporal lobe and the hippocampus. In: The Oxford Handbook of Memory (eds. E. Tulving and F.I.M. Craik), pp. 501-520. Oxford University Press, Oxford.

Received December 22, 2005; accepted in revised form January 3, 2006. 


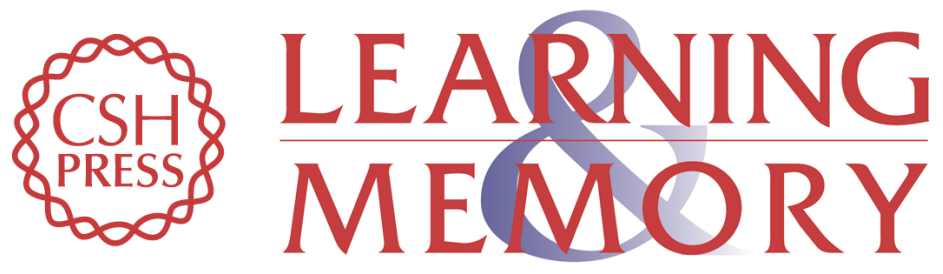

\section{Lack of evidence for a role of medial temporal lobe structures in visual perception}

Larry R. Squire, Yael Shrager and Daniel A. Levy

Learn. Mem. 2006, 13:

Access the most recent version at doi:10.1101//m.178406

References This article cites 10 articles, 2 of which can be accessed free at:

http://learnmem.cshlp.org/content/13/2/106.full.html\#ref-list-1

License

Email Alerting Receive free email alerts when new articles cite this article - sign up in the box at the Service top right corner of the article or click here. 